\title{
Simultaneous Control and \\ Piezoelectric Insert Optimization for Manipulators with Flexible Link
}

Valdecir Bottega

vbottega@ucs.br

Rejane Pergher

rpergher@ucs.br

University of Caxias do Sul - UCS

Dep. of Mathematics and Statistic 95070-560 Caxias do Sul, RS, Brazil

\author{
Jun S. O. Fonseca \\ jun@ufrgs.br \\ Federal Univ. of Rio Grande do Sul - UFRGS \\ Dep. of Mechanical Engineering \\ 90050-170 Porto Alegre, RS, Brazil
}

This work proposes a tracking control model for a flexible link robotic manipulator using simultaneously motor torques and piezoelectric actuators. The dynamic model of manipulator is obtained in a closed form through the Lagrangian approach. The control uses the motor torques for the tracking control of the joints and also to reduce the low frequency vibration induced in the manipulator links. The stability of this control is guaranteed by the Lyapunov stability theory. Piezoelectric actuators and sensors are added for controlling the high frequency vibrations beyond range of motor torque control. Additionally, this work introduces a formulation for simultaneous control and piezoelectric inserts geometry optimization through the maximization of the control action dissipated energy. Simulations on Matlab/Simulink are used to verify the efficiency of the control model.

Keywords: piezoelectric actuators, flexible links, tracking control, optimization, manipulators

\section{Introduction}

The design of light weight robotic manipulators with flexible links requires a control system which takes into account the interaction of the joint angles and the elastic deformation. This complex task has the additional complication of the essential uncertainty that characterizes robotic manipulators, such as variable payload and joint frictional torques (Siciliano and Valavanis, 1998).

A flexible robot control design is composed by two steps: a tracking control acting on the joint angles and a stabilizer for motion induced vibration suppression (Zhang et al., 2005; Mohamed et al., 2005).

Robotic systems can be considered linear with respect to some parameters, such as mass, inertia and damping factors, but this assumption is not valid for the state. Therefore, a position control law must be defined with an appropriate tracking error asymptotic stability, obtained with Lyapunov functions (Arimoto, 1996).

In this work, we propose a tracking control model for a robot arm with flexible link. Motor torques tracking control based on the elastic links dynamic reduces the low frequency vibrations. However, the high frequency modes cannot be eliminated by the motor torque action alone, because the torque control system low speed is unsuited to control high frequency vibrations. Thus, high frequency vibrations control must use faster response actuators like piezoelectrics. The actuation frequency ranges of the motor and the piezoelectrics inserted are chosen to be non-overlapping, so that their controls are uncoupled. Similar techniques have been already proven in single-link flexible manipulators (Sun et al., 2004; Choi and Shin, 1996; Cho et al., 1999) and in two-link flexible manipulators in (Kim et al., 2001).

The lower fundamental modes are responsible for most of the tip displacement of the robot arm, therefore only the first two eigenfunctions are considered here. The theory formulated in this work can be used for more than one flexible link, but for simplification, the simulated model has one rigid and one flexible link.

Robotic manipulator design has largely ignored the latest structural design methodologies. There is ground for improving robotic manipulators using modern structural optimization techniques, including the use of smart materials to assist the control

Paper accepted January, 2009. Technical Editor: Domingos A. Rade by changing the structural behavior (Banks et al., 1996; Gabbert and Schulz, 1996).

In this work, we propose a tracking control model for a planar robot with a flexible link. The motor torque controls the joint angle tracking and decreases the low frequency link vibration, while piezoelectric sensor and actuator are added to control the high frequency vibration. Furthermore, we propose a location and sizing optimization where the mass and stiffness changes due to the addition of sensors and actuators included. This has been already used in suppressing vibrations in a flexible beam system (Abreu et al., 2003; Dhuri and Seshu, 2006; Kermani et al., 2004). A Matlab/Simulink code was created to assess the control model efficiency.

$$
\begin{aligned}
& \text { Nomenclature } \\
& a_{i} \quad=\text { length of link } i \text {. } \\
& A_{b} \quad=\text { cross section of the link. } \\
& b \quad=\text { width of the flexible link. } \\
& b_{i j}=\text { inertia matrix terms. } \\
& c_{i j}=\text { Coriolis and centrifugal effects matrix terms. } \\
& B(q)=\text { positive definite symmetric inertia matrix. } \\
& C_{a} \quad=\text { input piezoelectric control matrix. } \\
& C(q, \dot{q}) \dot{q}=\text { Coriolis and centrifugal forces vector. } \\
& c_{s} \quad=\text { capacitance of the film sensor. } \\
& C_{k, i j}=\text { constants. } \\
& D=\text { positive semidefinite link damping diagonal matrix. } \\
& D_{\Delta} \quad=\text { positive definite gain diagonal matrix. } \\
& d_{31}=\text { piezoelectric constant } \text {. } \\
& d_{n i}=\text { distance from the bottom of the piezofilm sensor to the } \\
& \text { neutral axis. } \\
& d_{k y i}\left(x_{i}, t\right)=\text { deflection of flexible link. } \\
& E_{b} \quad=\text { elastic modulus of the flexible link. } \\
& E_{c} \quad=\text { elastic modulus of the piezoceramic actuator. } \\
& E_{f} \quad=\text { elastic modulus of the piezofilm sensor } \text {. } \\
& (E I)_{i}=\text { flexural stiffness property of the link } i \text {. } \\
& f_{i j} \quad=\text { deflection velocity depend functions. } \\
& g(q)=\text { gravitational torque vector. }
\end{aligned}
$$


$g_{31}=$ piezoelectric stress constant .

$K_{c} \quad=$ piezoelectric feedback gain matrix.

$K_{e} \quad=$ positive definite stiffness diagonal matrix.

$K_{p} \quad=$ positive definite control gain diagonal matrix.

$K_{\text {piez }}=$ modal stiffness due to the actuator and sensor .

$K_{31}^{2}=$ electromechanical coupling factor .

$l_{a} \quad=$ length of the piezoelectric actuator and sensor.

$m_{i} \quad=$ link mass.

$m_{h i}=$ motor mass.

$q=[\theta, \delta]^{T}=$ generalized coordinates vector .

$\dot{q}_{r} \quad=$ reference velocity vector.

$\tilde{q} \quad=$ tracking error vector .

$q_{d} \quad=$ desired path vector .

$s \quad=$ reference error vector .

$t_{b} \quad=$ link thickness.

$t_{c} \quad=$ piezoceramic thickness.

$t_{f} \quad=$ piezofilm thickness.

$u \quad=$ input control torque vector.

$V_{f}(t)=$ voltage generated by the piezofilm sensor .

$V_{a}(t)=$ input voltage to the piezoelectric actuator.

$x_{a} \quad=$ location of the actuator on the link.

$W\left(t_{0}\right)=$ initial energy of the system.

$W_{f} \quad=$ energy dissipated by internal damping.

$W_{c} \quad=$ energy dissipated by the control action.

\section{Greek Symbols}

$\alpha=$ constant of material cost .

$\beta_{i j} \quad=$ constant of feedback gain.

$\delta \quad=n \times 1$ elastic modes coordinates vector.

$\delta_{d} \quad=n \times 1$ desired elastic modes coordinates vector.

$\varepsilon_{i j}, \varphi_{i j}=$ positive constants.

$\phi_{k i j}=$ eigenfunctions.

$\lambda_{i} \quad=$ weight elements.

$\bar{\omega}_{i j} \quad=j$ th natural angular frequency.

$\theta=n \times 1$ joint coordinates vector.

$\rho_{b} \quad=$ beam mass density .

$\rho_{k i} \quad=$ link $i$ uniform density.

$\tau \quad=$ input motor torque.

$\zeta \quad=$ modal damping.

$\Lambda \quad=$ gain diagonal matrix.

\section{Dynamic Model}

The control laws are obtained from the arm motion equations. Figure 1 shows a simplified planar manipulator composed by one rigid and one flexible link, two joints, two motors, one piezoelectric actuator and one sensor attached to the top side and bottom side of the flexible link, respectively.

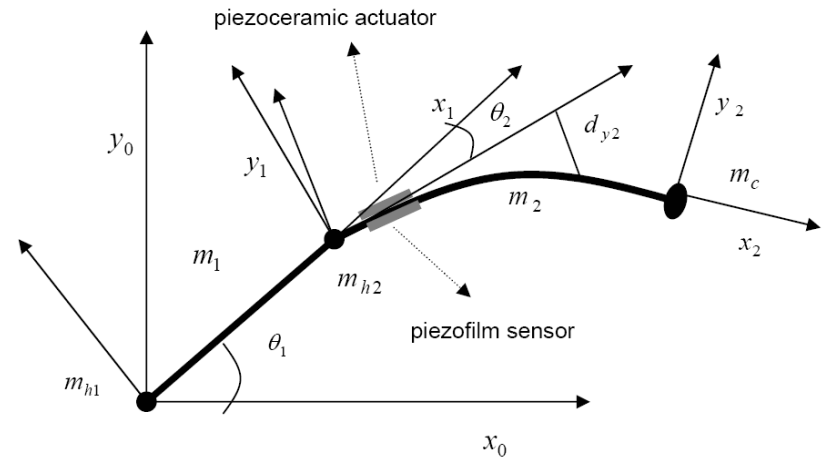

Figure 1. Model of planar manipulator with one rigid and one flexible link featuring piezoelectric actuator and sensor.

The motion of the robot endpoint is a composition of the successive relative link motions. This movement is described using homogeneous matrix transformations. These transformations represent translations and rotations due to the joints angle changes and the flexible link elastic deflections (Book, 1984; Bottega et al., 2007). The deflections are obtained considering each link as an uniform beam with length $a_{i}$ featuring a piezoceramic actuator bonded to the top face, and a piezofilm sensor bonded to the bottom face as shown in Fig. 2.
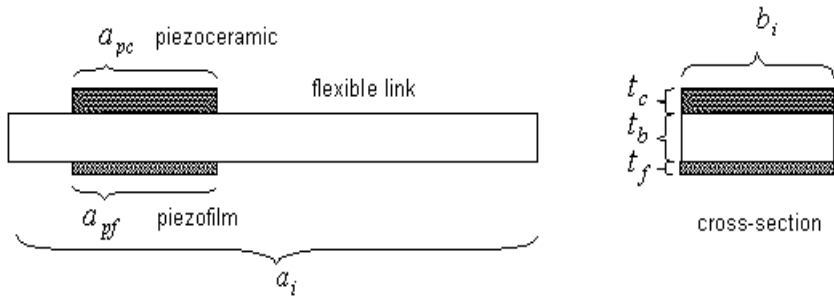

cross-section

Figure 2. A flexible link of manipulator featuring surface-bonded piezoelectric actuator and sensor.

Flexible links featuring surface-bonded piezoelectric actuator and sensor can be modeled as discontinuous cross-section EulerBernoulli beams, with deflection $d_{k y i}(x, t)$, satisfying the partial differential equation system (Tsukazan, 2005; Copetti et al., 2007)

$$
\begin{aligned}
& \left(E I_{1}\right)_{i} \frac{\partial^{4} d_{1 y i}(x, t)}{\partial x^{4}}+\rho_{1 i} \frac{\partial^{2} d_{1 y i}(x, t)}{\partial t^{2}}=0,0 \leq x<x_{a} \\
& \left(E I_{2}\right)_{i} \frac{\partial^{4} d_{2 y i}(x, t)}{\partial x^{4}}+\rho_{2 i} \frac{\partial^{2} d_{2 y i}(x, t)}{\partial t^{2}}=0, x_{a} \leq x<x_{a}+l_{a} \\
& \left(E I_{3}\right)_{i} \frac{\partial^{4} d_{3 y i}(x, t)}{\partial x^{4}}+\rho_{3 i} \frac{\partial^{2} d_{3 y i}(x, t)}{\partial t^{2}}=0, x_{a}+l_{a} \leq x \leq a_{i}
\end{aligned}
$$

where $\rho_{k i}$ is the uniform density, $\left(E I_{k}\right)_{i}$ is the flexural rigidity property of the link i (Meirovitch, 1967), and the index k selects the position along the beam length, where 1,2 , and 3 mean respectively before, within, and after the piezoelectric actuator.

Exploring the time and space separability of the system Eq. (1) by the modal analysis technique (Knani, 2002), the link $i$ deflection can be expressed as 


$$
d_{k y i}(x, t)=\sum_{j=1}^{m} \phi_{k i j}(x) \delta_{i j}(t)
$$

where each term in the general solution of Eq. (1) is the product of a time harmonic function of the term $\delta_{i j}=e^{j \varpi_{i j} t}$ and of a space eigenfunction of the form

$$
\begin{aligned}
\phi_{k i j}(x) & =C_{1, i j} \sin \left(\beta_{i j} x\right)+C_{2, i j} \cos \left(\beta_{i j} x\right) \\
& +C_{3, i j} \sinh \left(\beta_{i j} x\right)+C_{4, i j} \cosh \left(\beta_{i j} x\right)
\end{aligned},
$$

where $\beta_{i j}{ }^{4}=\varpi_{i j}{ }^{2} /(E I)_{i}$ and $\varpi_{i j}$ is the jth vibration mode angular velocity for the link $\mathrm{i}$, derived from an eigenvalue problem. The determination of the constant coefficients $C_{k, i j}$ uses clamped conditions at the link base and mass boundary conditions representing the balance of bending moment, shearing force at the link endpoint (De Luca et al., 1988), and the internal boundary conditions are expressed in Table 1.

Table 1. Discontinuity conditions

\begin{tabular}{|l|l|}
\hline First discontinuity & Second discontinuity \\
\hline$\phi_{1 i j}\left(x_{a}\right)=\phi_{2 i j}(0)$ & $\phi_{2 i j}\left(l_{a}\right)=\phi_{3 i j}(0)$ \\
\hline$\phi^{\prime}{ }_{1 i j}\left(x_{a}\right)=\phi^{\prime}{ }_{2 i j}(0)$ & $\phi^{\prime}{ }_{2 i j}\left(l_{a}\right)=\phi_{3 i j}^{\prime}(0)$ \\
\hline$\phi^{\prime \prime}{ }_{1 i j}\left(x_{a}\right)=\alpha^{4} \phi^{\prime \prime}{ }_{2 i j}(0)$ & $\phi^{\prime \prime}{ }_{2 i j}\left(l_{a}\right)=\alpha^{4} \phi^{\prime \prime}{ }_{3 i j}(0)$ \\
\hline$\phi^{\prime \prime \prime}{ }_{1 i j}\left(x_{a}\right)=\alpha^{4} \phi^{\prime \prime \prime}{ }_{2 i j}(0)$ & $\phi^{\prime \prime \prime}{ }_{2 i j}\left(l_{a}\right)=\alpha^{4} \phi^{\prime \prime \prime}{ }_{3 i j}(0)$ \\
\hline
\end{tabular}

Starting from this analysis, the mode shape for flexible link is

$$
\phi_{i j}(x)=\left\{\begin{array}{l}
\phi_{1 i j}(x), \quad 0 \leq x<x_{a} \\
\phi_{2 i j}\left(x-x_{a}\right), \quad x_{a} \leq x<x_{a}+l_{a} \\
\phi_{3 i j}\left(x-\left(x_{a}+l_{a}\right)\right), \quad x_{a}+l_{a} \leq x \leq a_{i}
\end{array}\right.
$$

where $l_{a}$ and $x_{a}$ are the length and the location of the piezoceramic actuator and the piezofilm sensor respectively as shown in Fig. 3.

\section{Equations of Motion}

The equations of motion are derived in closed form using a Lagrangian approach written in the form of compact matrices, resulting (Book, 1984):

$$
B(q) \ddot{q}+C(q, \dot{q}) \dot{q}+K_{e} q+D \dot{q}+g(q)=u,
$$

where $q=[\theta, \delta]^{T}$ is the generalized coordinates vector.

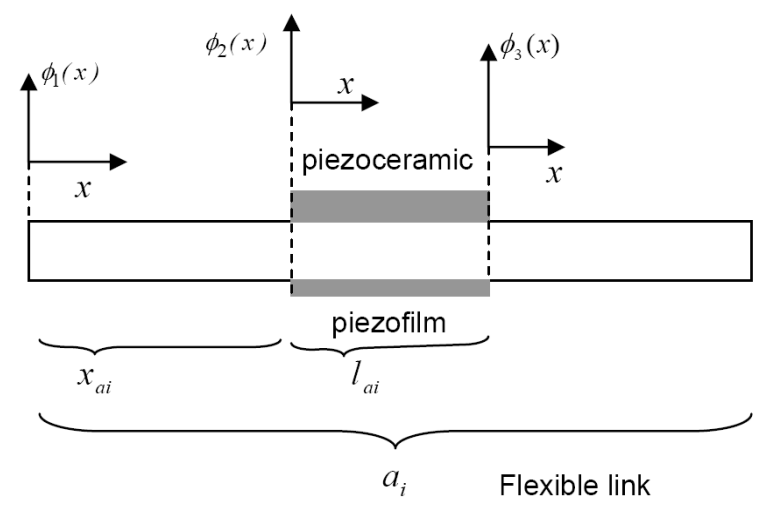

Figure 3. A flexible link of manipulator featuring surface-bonded piezoelectric actuator and sensor.

The matrices of the dynamic model can be partitioned as

$$
\begin{aligned}
& B(q)=\left[\begin{array}{ll}
B_{\theta \theta} & B_{\theta \delta} \\
B_{\theta \delta}^{T} & B_{\delta \delta}
\end{array}\right], \quad C(q, \dot{q})=\left[\begin{array}{ll}
C_{\theta \theta} & C_{\theta \delta} \\
C_{\delta \theta} & C_{\delta \delta}
\end{array}\right], \quad K_{e}=\left[\begin{array}{ll}
0 & 0 \\
0 & K
\end{array}\right] \\
& g(q)=\left[\begin{array}{l}
g_{\delta}(\theta) \\
g_{\theta}(\delta)
\end{array}\right], \quad u=\left[\begin{array}{l}
\tau \\
0
\end{array}\right],
\end{aligned}
$$

where the indexes $\theta \theta, \theta \delta$ and $\delta \delta$ are the terms from the matrices corresponding to rigid body, rigid coupling with flexible body and flexible body, respectively.

\section{Tracking Control}

This section introduces the flexible robot arm tracking control, based on an adaptive controller presented by Arteaga and Siciliano, (2000) and a robust control law to reduce the elastic vibrations of the arms. The stability of the tracking error is proved using the Lyapunov stability theory (La Salle and Lefschetz, 1961). The improved tracking controller using nominal compensation of dynamic nonlinearities of system Eq. (5) is given by

$$
u=B(q) \ddot{q}_{r}+C(q, \dot{q}) \dot{q}_{r}+K_{e} q_{d}+D \dot{q}_{r}+g(q)-K_{p} s,
$$

where $\dot{q}_{r}=\dot{q}_{d}-\Lambda \tilde{q}$ is the reference velocity vector with tracking error $\tilde{q}=q-q_{d}$ and $s=\dot{q}-\dot{q}_{r}=\dot{\tilde{q}}+\Lambda \tilde{q}$ is the reference error.

Inserting Eq. (7) in the dynamic equation, Eq. (5), the error equation in $s$ becomes

$$
B(q) \dot{s}=-\left(C(q, \dot{q}) s+K_{e} \tilde{q}+D s+K_{p} s\right)
$$

In order to prove the stability at the origin of Eq. (8), consider $x=\left[\begin{array}{lll}\tilde{q} & \dot{\tilde{q}}\end{array}\right]^{T}$ with the Lyapunov function:

$$
V(x, t)=V(x)=\frac{1}{2} s^{T} B(q) s+\tilde{q}^{T}\left(\Lambda K_{p}+K_{e}\right) \tilde{q}
$$

Deriving Eq. (9) along Eq. (8), using the property of dynamic equation that $B(q)-2 C(q, \dot{q})$ is skew symmetric and $s^{T}(B(q)-2 C(q, \dot{q})) s=0$ (Arteaga, 1998$)$, we have

$$
\dot{V}(x)=-\dot{\tilde{q}}^{T} K_{p} \dot{\tilde{q}}-\tilde{q}^{T}\left(\Lambda K_{p} \Lambda+\Lambda K_{e}\right) \tilde{q}
$$


Since $K_{p}, \Lambda$ and $K_{e}$ are positive definite and diagonal matrices, $\dot{V}(x) \leq 0$ which implies that the equilibrium point $x=0$ is asymptotically stable (Arteaga and Siciliano, 2000). However, it is not guaranteed that the deflections tend to zero for weakly damped system. In this case, we can add a robust control law (Arimoto, 1996) that damps the system and eliminates the steady vibrations as

$$
\begin{aligned}
& D^{\prime}{ }_{\Delta} \dot{\delta}_{d}=\left(D_{\Delta}-\operatorname{diag}\left\{f_{11}, \ldots, f_{1 r_{1}}, \ldots, f_{n r_{1}}, \ldots, f_{n r_{n}}\right\}\right) \dot{\delta}_{d} \\
& f_{i j}=\frac{\dot{\delta}_{d i j} s_{d i j}}{\left\|\dot{\delta}_{d i j} s_{d i j}\right\|+\varepsilon_{i j} e^{-\varphi_{i j}}} \quad i=1, \ldots, n \quad j=1, \ldots, r_{i},
\end{aligned}
$$

where $\dot{\delta}_{d i j}, s_{d i j}$ are generic elements dependent on desired deflections $\delta_{d}$ and tracking error $s, r_{i}$ is the number of deflection generalized coordinates for link $i$, and $n$ is the number of links. The robust control law Eq. (11) presents strong adaptation to various perturbation from modeling errors and disturbance, and guaranteed transient performance (Yao and Tomizuka, 1996).

To prove the stability of the deflections $\delta_{i}$ from Eqs. (5) and (6), and assuming that $\theta_{d}$ is constant, we take the partitioned equation, for a desired deflection $\delta_{d}$ :

$$
B_{\delta \delta} \ddot{\delta}_{d}+C_{\delta} \delta_{d}+D_{\Delta}^{\prime} \dot{\delta}_{d}+K \delta_{d}+g\left(\delta_{d}\right)=0
$$

and we consider $y=\delta_{d}+K^{-1} g_{\delta}$. Substituting in Eq. (12) we have

$$
B_{\delta \delta} \ddot{y}+C_{\delta} \dot{y}+D_{\Delta}^{\prime} \dot{y}+K y=0
$$

with Lyapunov function

$$
V_{y}(y, \dot{y})=\frac{1}{2} y^{T} K y+\frac{1}{2} \dot{y}^{T} B_{\delta \delta} \dot{y}
$$

The time derivative of Eq. (14) along Eq. (13) using the property of dynamic equation that $B_{\delta \delta}-2 C_{\delta}$ is skew symmetric and $y^{T}\left(B_{\delta \delta}-2 C_{\delta}\right) y=0,($ Arteaga, 1998) results in

$$
\dot{V}_{y}(y, \dot{y})=-\dot{y}^{T} D_{\Delta}^{\prime} \dot{y}
$$

Since $D^{\prime}{ }_{\Delta}$ is a diagonal positive definite matrix, $\dot{V}_{y}(y, \dot{y}) \leq 0$ which implies that the equilibrium point $y=0$ is asymptotically stable.

The Eq. (11) is added to Eq. (7) to obtain the control law of the system Eq. (4) expressed as

$$
\begin{aligned}
& u=B(q) \ddot{q}_{r}+C(q, \dot{q}) \dot{q}+K_{e} q_{d}+D \dot{q}_{r} \\
& +g(q)-K_{p} s+\left[0^{T} \quad\left(D_{\Delta}^{\prime} \dot{\delta}_{d}\right)^{T}\right]^{T}
\end{aligned}
$$

The proof of the stability of this control law can be obtained using Lyapunov stability theory in a manner similar to that shown in the stability of the control law Eq. (7). So the control law Eq. (16) is stable on the origin and tracking error $\tilde{q}$ tends to zero. The damping of the system has been increased, and the deflection modes tend to zero. A detailed proof of the stability of this control law can be seen in Arteaga and Siciliano (2000).

\section{Piezoelectric Sensors and Actuators Control}

Under certain conditions, achieving the suppression of elastic link vibrations by means of the motor torque alone may be very difficult. Hardware limitations, such as motor saturation and motor noise may prevent the control of high frequency vibration modes. To solve these problems we propose a hybrid controller consisting of the servo-motor and piezoelectric actuators and sensors bonded to the flexible links (Shin and Choi, 2001; Liu and Begg, 2000). We obtain a controller that relies on the motor torque to achieve the desired path and a feedback voltage control on the piezoelectric actuators for the elastic vibrations.

We propose a feedback control voltage to the piezoceramic actuator (Crawley and De Luis, 1987), expressed as

$$
V_{a}(t)=-C_{a}^{T} K_{c} \dot{V}_{f}(t)
$$

With

$$
C_{a}=\frac{E_{b} E_{c} t_{c} t_{f} d_{31}}{\rho_{b} A_{b}\left(E_{b} t_{b}+6 E_{c} t_{c}\right)}\left(\phi^{\prime}\left(x_{a}+l_{a}\right)-\phi^{\prime}\left(x_{a}\right)\right)
$$

The voltage generated by the piezofilm sensor $V_{f}(t)$ obtained by integrating the electric charge, developed at a point on the piezofilm is expressed as (Banks et al., 1996)

$$
V_{f}(t)=c_{s} \delta=\frac{k_{31}^{2} b_{f}}{C g_{31}} d_{n i} \delta
$$

This additional controller Eq. (17) is combined to the original one, Eq. (16). The resulting control law for the system Eq. (5) is expressed as

$$
\begin{aligned}
& u=B(q) \ddot{q}_{r}+C(q, \dot{q}) \dot{q}+K_{e} q_{d}+D \dot{q}_{r} \\
& +g(q)-K_{p} s+\left[\begin{array}{ll}
0^{T} & \left(D_{\Delta}^{\prime} \dot{\delta}_{d}\right)^{T}+C_{a} V_{a}(t)
\end{array}\right]^{T}
\end{aligned}
$$

resulting in a hybrid control act, where the motor torque controls the joint angle tracking and reduces the low frequency vibrations on the links while piezoelectric sensors and actuators are added to control the high frequency vibrations. Again, the proof of the stability of this control law can be obtained using Lyapunov stability theory as shown previously for the control law Eq. (7). So, the control law Eq. (20) is stable in the origin and tracking error $\tilde{q}$ tends to zero.

\section{Location and Sizing Actuators Optimization}

Controlling structural vibration depends not only on the control law, but also on the selection and location of the actuators and sensors (Denoyer and Kwak, 1996). In this work, we propose a methodology for the actuator and sensor position and sizing optimization, based on maximization of dissipation of the energy control ( $\mathrm{Li}$ et al., 2002). This procedure takes into account the actuators and sensors mass and stiffness and their effect on the mechanical behavior of the structure. This influence is combined to the control characteristics to obtain an objective function that depends on the actuators location and sizing, and the control gain. 
The deflections are obtained considering each link as a uniform beam with $a_{i}$ length featuring a piezoceramic actuator bonded to the top face and a piezofilm sensor bonded to the bottom face as shown in Fig. 3.

The dynamic of the flexible link with $m$ piezoelectric sensors and actuators in terms of modal coordinates can be expressed as

$$
B_{\delta \delta} \ddot{\delta}+C_{\delta \delta} \dot{\delta}+D \dot{\delta}+K \delta=C_{a} V_{a}(t)
$$

where $K=K_{e}+K_{\text {piez }}$ and $K_{\text {piez }}$ represent the modal stiffness due to the actuator and piezoelectric sensor.

The total energy stored in the system (De Luca et al., 1988) can be expressed as

$$
W=T+U=\frac{1}{2} \dot{\delta}^{T} B_{\delta} \dot{\delta}+\delta^{T} K \delta
$$

Differentiating the Eq. (22) with respect to the time, we obtain

$$
\dot{W}=\dot{T}+\dot{U}=\frac{1}{2} \dot{\delta}^{T} \dot{B}_{\delta \delta} \ddot{\delta}+\dot{\delta}^{T} B_{\delta \delta} \ddot{\delta}+\dot{\delta}^{T} K \delta
$$

Isolating $B_{\delta \delta} \ddot{\delta}$ on Eq. (21), replacing in Eq. (23) with the control law Eq. (17), and using the property of dynamic equation that $B_{\delta \delta}-2 C_{\delta \delta}$ is skew symmetric and $\delta^{T}\left(B_{\delta \delta}-2 C_{\delta \delta}\right) \delta=0$, we obtain

$$
\dot{W}=\dot{T}+\dot{U}=-\dot{\delta}^{T} D \dot{\delta}--\dot{\delta}^{T}\left(C_{a} K_{c} C_{a}{ }^{T} c_{s}\right) \dot{\delta},
$$

where the first and the second terms describe the energy rates removed from the system by the internal damping and by the control feedback, respectively.

Integrating the Eq. (24), we obtain

$$
W\left(t_{0}\right)=W_{f}+W_{c}=\int_{t_{0}}^{\infty} \dot{\delta}^{T} D \dot{\delta} d t+\int_{t_{0}}^{\infty} \dot{\delta}^{T}\left(C_{a} K_{c} C_{a}^{T} c_{s}\right) \dot{\delta} d t
$$

For effective vibration suppression, it is reasonable to derive a method to increase the energy dissipated by the control. We observe that $W_{c}$ depends on the locations $x_{a}$ and the length $l_{a}$ of the actuators, and feedback matrix gain $K_{c}$. Therefore $W_{c}$ can be used as an optimization criterion to determine location and sizing of actuator and feedback gains.

To determining $W_{c}$, we write the Eq. (21) in state-space form as

$$
\dot{z}=\tilde{H} z
$$

where $z=[\delta, \dot{\delta}]^{T}$ and

$$
\tilde{H}=\left[\begin{array}{cc}
0 & I \\
-B_{\delta \delta}^{-1} K & -B_{\delta \delta}{ }^{-1}\left(C_{\delta \delta}+D+C_{a} K_{c} C_{a}{ }^{T} c_{s}\right)
\end{array}\right] .
$$

The control-induced energy dissipation by the active damping control $W_{c}$ can be written as

$$
W_{c}=\int z^{T} Q z d t
$$

where

$$
Q=\left[\begin{array}{cc}
\mathbf{0} & \mathbf{0} \\
\mathbf{0} & C_{a} K_{c} C_{a}{ }^{T} c_{s}
\end{array}\right]
$$

is a $2 m \times 2 m$ matrix positive semi-definite, which allows proving the asymptotic stability using the Lyapunov theory of the Eq. (26) system (Naidu, 2003).

Applying transformation technique to the Eq. (28), we obtain (Truhar and Veselić, 2004)

$$
W_{c}=z^{T} P z
$$

where $P$ is symmetric positive definite matrix, solution of the Lyapunov equation

$$
\tilde{H} P+P \tilde{H}=-Q
$$

It is noticeable that $W_{c}$ depends on the initial conditions of the flexible structure. In order to eliminate this dependence, we assume that the initial state of $z$ satisfies $W_{a}^{-1} z_{0}$ where

$$
W_{a}=\left[\begin{array}{cc}
M & 0 \\
0 & M
\end{array}\right] \text { and } M=\left[\begin{array}{cccc}
\lambda_{1} & & & \\
& \lambda_{2} & & \\
& & \ddots & \\
& & \lambda_{n}
\end{array}\right]
$$

with random value of $\lambda_{i}>0$ where $\lambda_{1}$ and $\lambda_{2}$ are larger than the others, because it is expected that the lowest frequency modes are more easily excited by the rigid body motion (Li et al., 2002). Therefore, we obtain an objective function (Truhar, 2004),

$$
J_{0}=\operatorname{tr}\left(W_{a}^{T} P W_{a}\right)
$$

for energy dissipated by the control which depends on the location $x_{a}$, the length $l_{a}$ of the piezoelectric actuators and the gain $K_{c}$.

To design a precise and agile manipulator, it is reasonable to take it as light as possible. This is accomplished by adding a function of the actuator and sensor masses to the objective function shown above. We added to $J_{0}$ a quadratic dependency on the actuator length $l_{a}$ (Li et al., 2002), resulting the following composite objective function:

$$
\min _{x_{a}, l_{a}, K_{c}} J(\alpha)
$$

with

$$
\begin{aligned}
& J(\alpha)=\alpha l_{a}^{2}-J_{0} \\
& 0 \leq x_{a} \leq a_{i} \\
& 0<l_{a}+x_{a} \leq a_{i} \\
& K_{c} \leq K_{\max }
\end{aligned}
$$

where $\alpha$ depends on the piezoelectric material cost and $K_{\max }$ depends on the actuator power limitation.

\section{Results}

The control laws were tested on a simplified robot model with two links: the first one is rigid and the second one is flexible with two deformation modes, as shown in Fig. 1. Gravitational effects were ignored (De Luca et al., 1990). The Lagrangian coordinate vector is $q=\left(\theta_{1}, \theta_{2}, \delta_{21}, \delta_{22}\right)$ and the inertial matrix and the Coriolis and centrifugal effects matrix are 


$$
\begin{aligned}
& b_{11}=I_{h 1}+h_{1}+2 h_{2}+m_{2} l_{2}^{2}+m_{h 2} a_{1}^{2}+I_{2}+I_{h 2}, \\
& b_{12}=b_{21}=h_{1}+h_{2}+m_{2} l_{2}^{2}+I_{l 2}+I_{h 2}, \\
& b_{13}=b_{31}=b_{14}=b_{41}=m_{2} a_{1} c\left(\theta_{2}\right)+m_{2} l_{2}, \\
& b_{22}=h_{1}+m_{2} l_{2}^{2}+I_{l 2}+I_{h 2} ; b_{23}=b_{32}=b_{24}=b_{42}=m_{2} l_{2}, \\
& b_{33}=b_{43}=b_{44}=m_{2}, \\
& c_{21}=h_{3} \dot{\theta}_{1}-\frac{1}{2} h_{3} \dot{\theta}_{2}+\frac{1}{2} m_{2} a_{1} s_{2} \dot{\delta}_{1}+\frac{1}{2} m_{2} a_{1} s_{2} \dot{\delta}_{2}, \\
& c_{11}=-2 h_{3} \dot{\theta}_{2} ; c_{12}=-h_{3} \dot{\theta}_{2} ; c_{22}=\frac{1}{2} h_{3} \dot{\theta}_{1} ; c_{31}=c_{41}=-m_{2} a_{1} s_{2} \dot{\theta}_{2}, \\
& c_{32}=c_{42}=c_{33}=c_{43}=c_{34}=c_{44}=0,
\end{aligned}
$$

where

$$
\begin{aligned}
& h_{1}=m_{2} d_{y}^{2} ; h_{2}=m_{2} a_{1} s_{2} d_{y 2}^{2} ; h_{3}=m_{2} a_{1} c_{2} d_{y 2}^{2}+m_{2} a_{1} s_{2} l^{2}, \\
& s_{i}=\sin \left(\theta_{i}\right) ; c_{i}=\cos \left(\theta_{i}\right) ; d_{y 2}=\phi_{21} \delta_{21}+\phi_{22} \delta_{22},
\end{aligned}
$$

while the stiffness and modal damping matrices are

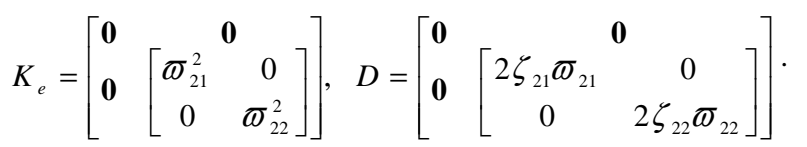

The following diagonal elements of gain matrices for the control are

$$
\begin{aligned}
& K_{p 11}=0 ; K_{p 22}=0 ; K_{p 33}=90 ; K_{p 44}=14, \\
& \Lambda=D_{\Delta}=I_{4 \times 4}, \\
& K_{c 11}=0 ; K_{c 22}=0 ; K_{c 33}=30 ; K_{c 44}=30, \\
& \lambda_{1}=20 ; \lambda_{2}=10 .
\end{aligned}
$$

\section{Physical Parameters}

We present the mechanical and geometrical properties of the piezoelectric materials (Choi and Shin, 1996) used in this work:

$$
\begin{aligned}
& a_{1}=0,3 \mathrm{~m} ; a_{2}=0,7 \mathrm{~m} ; l_{1}=0,15 \mathrm{~m} ; l_{2}=0,35 \mathrm{~m}, \\
& m_{1}=0,2 \mathrm{~kg} ; m_{2}=0,3 \mathrm{~kg} ; m_{h 1}=m_{h 2}=1,0 \mathrm{~kg}, \\
& I_{1}=0,170 ; I_{2}=0,103 ; I_{h 1}=0,230 ; I_{h 2}=0,198\left(\mathrm{kgm}^{2}\right), \\
& \varpi_{21}=4,716.2 \pi \mathrm{rad} / \mathrm{sec} ; \Phi_{22}=14,395.2 \pi \mathrm{rad} / \mathrm{sec}, \\
& \zeta_{21}=0,07 ; \zeta_{22}=0,03 ; \varepsilon_{i j}=0,1 ; \varphi_{i j}=0,01, \\
& \phi_{21}=-1,446 ; \phi_{22}=1,369, \\
& \rho_{f}=1780 \mathrm{~kg} / \mathrm{m}^{3} ; \quad \rho_{2}=7700 \mathrm{~kg} / \mathrm{m}^{3} ; \\
& E_{c}=64 \mathrm{GPa} ; E_{f}=2 \mathrm{GPa} ; E_{b}=65 \mathrm{GPa} ; \\
& \mathrm{t}_{\mathrm{c}}=0.8 \mathrm{~mm} ; \mathrm{t}_{\mathrm{f}}=0.028 \mathrm{~mm} ; \mathrm{t}_{\mathrm{b}}=0.8 \mathrm{~mm} ; \mathrm{b}=25 \mathrm{~mm} ; \\
& \mathrm{d}_{31}=-300 \times 10^{-12}(\mathrm{~m} / \mathrm{m}) /(\mathrm{V} / \mathrm{m}) ; \mathrm{g}_{31}=216 \times 10^{-3}(\mathrm{~V} / \mathrm{m}) /\left(\mathrm{N} / \mathrm{m}^{2}\right) ; \\
& \mathrm{C}=380 \mathrm{pF} / \mathrm{cm}^{2} .
\end{aligned}
$$

The results were obtained using a block-diagram implemented in MatLab/Simulink software presented in Fig. 4, where the fourthorder Runge-Kutta method with $\Delta t=1 \mathrm{~ms}$ was used to integrate the equations for a five second simulation.

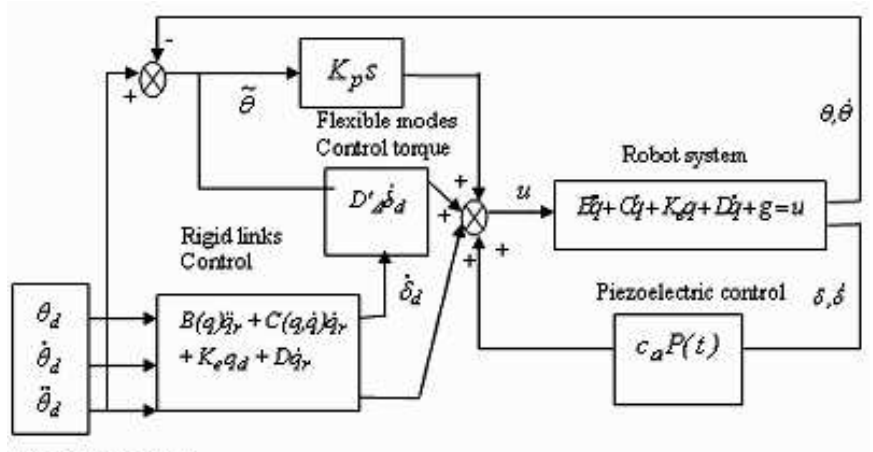

Desired trajectory

Figure 4. Block-diagram of the proposed control algorithm.

\section{Desired Trajectory}

Figure 5 shows the trapezoidal trajectory tracking used with amplitude $\pi / 2$ for the joint angles 1 and 2 without initial tracking error.

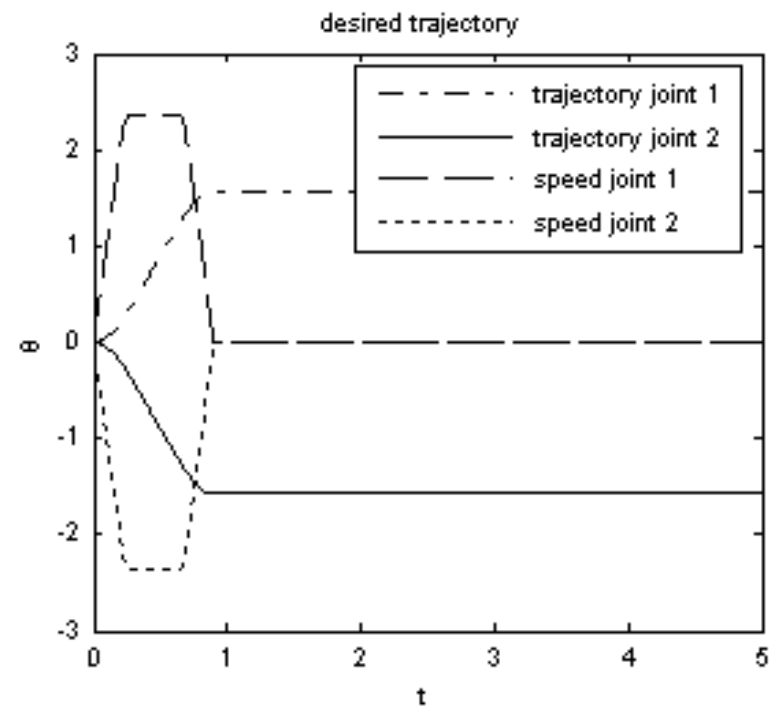

Figure 5. Desired trajectory of the joint angle 1 and 2.

Figure 6 shows a circle path selected as an end-point trajectory. The circle has $0.4 \mathrm{~m}$ of diameter. Equation (36) is executed in a clockwise direction starting at the top point of the circle in $x y$ plane.

$$
\begin{aligned}
& x=r \cos (t)+0,4 \\
& y=r \sin (t)
\end{aligned}
$$




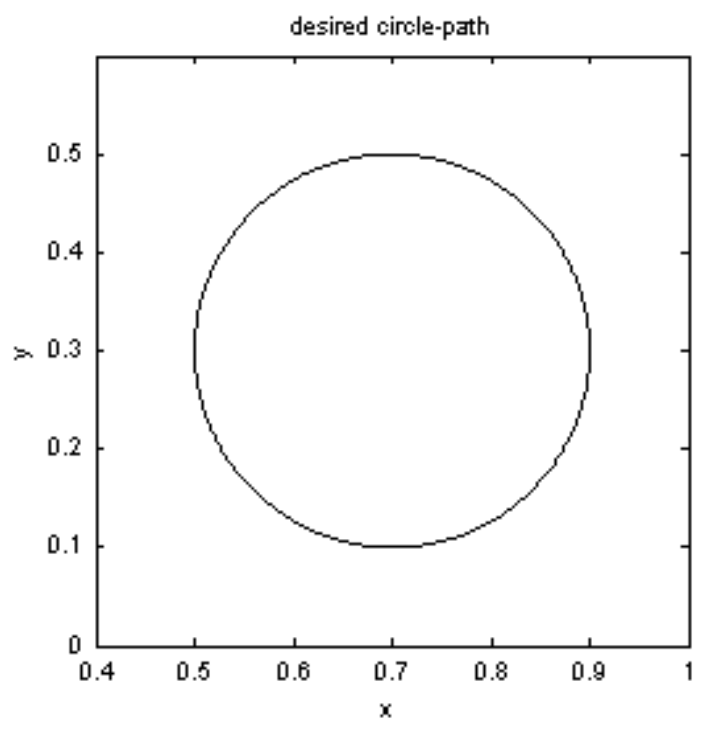

Figure 6. Desired circle-path, end-point trajectory.

Figure 7 shows the joint trajectories generated by inverse kinematics from the desired end-point motion.

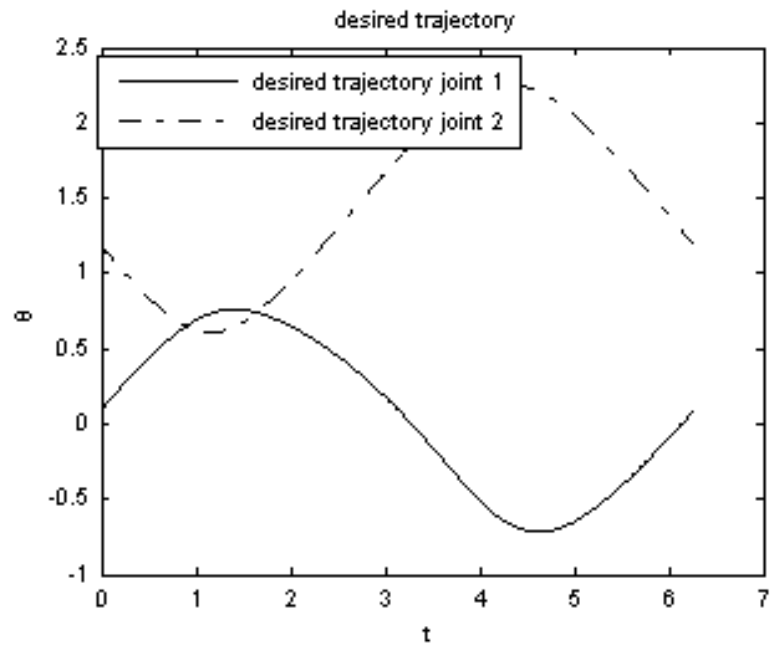

Figure 7. Desired joint angle trajectory for a circle-path, end-point trajectory.

\section{Performance Indices}

Performance measures commonly used such as the rising time, damping and steady state error are not adequate for nonlinear systems such as robot. In Yao and Tomizuka (1996), the valued $\mathrm{L}^{2}$ norm given by

$$
L^{2}[\delta(t)]=\left(\frac{1}{T_{f}} \int_{0}^{T_{f}}\|\delta(t)\|^{2} d t\right)^{1 / 2}
$$

is used as an objective numerical tracking measure performance for an entire curve $\delta(t)$. It is an average measure, and large deflections during the initial transient stage cannot be represented.

Also, we will use the average tracking error of joint $i$ during the last three seconds,

$$
L[\delta(t)]=\frac{1}{3} \int_{T_{f}-3}^{T_{f}} \delta(t) d t
$$

as indexes to measure the steady state tracking error.

\section{Simulations}

Firstly, we simulated a damped system with a control law, Eq. (7). Figure 8 shows that the elastic deflections tend to zero and they are limited due to structural damping of the system. Figure 9 shows that the system tracking error also tends to zero. In the second simulation, we used the control law given by Eq. (16) in the same system used before. Figure 10 shows an increase in the system damping and a faster convergence to zero of the deflections. This is a result of the addiction of $D_{\Lambda}^{\prime} \dot{\delta}_{d}$ controller.

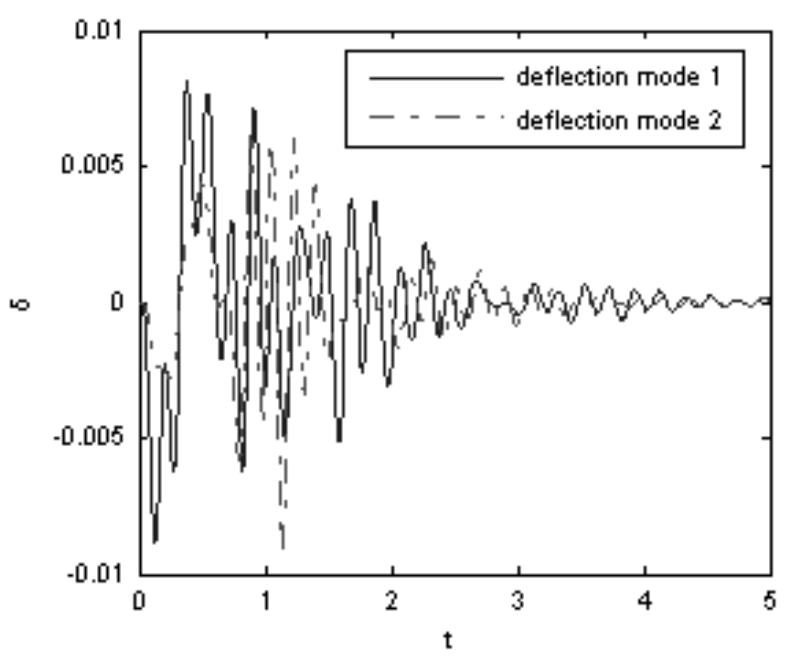

Figure 8. Deflection of modes 1 and 2 for the damped system.

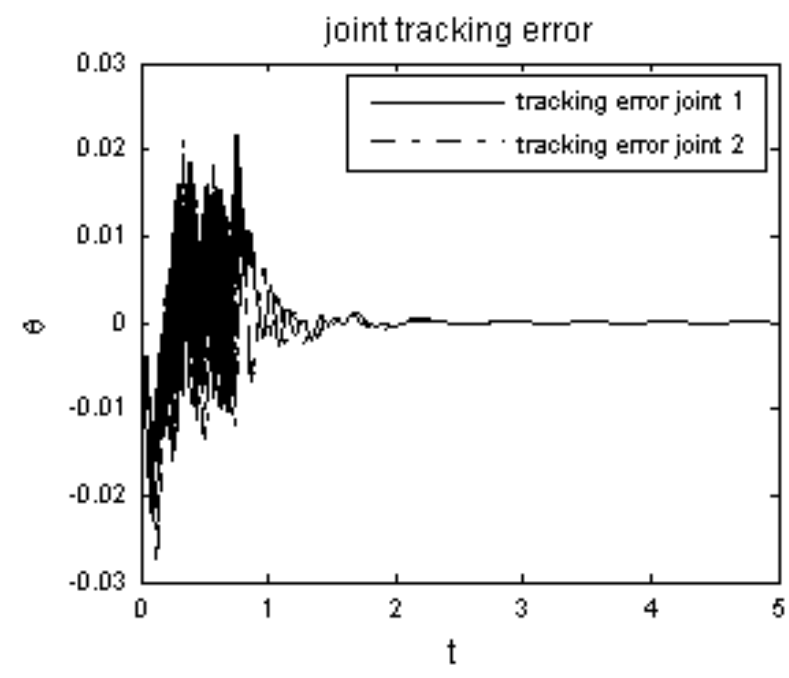

Figure 9. Joint tracking error of the trapezoidal trajectory. 


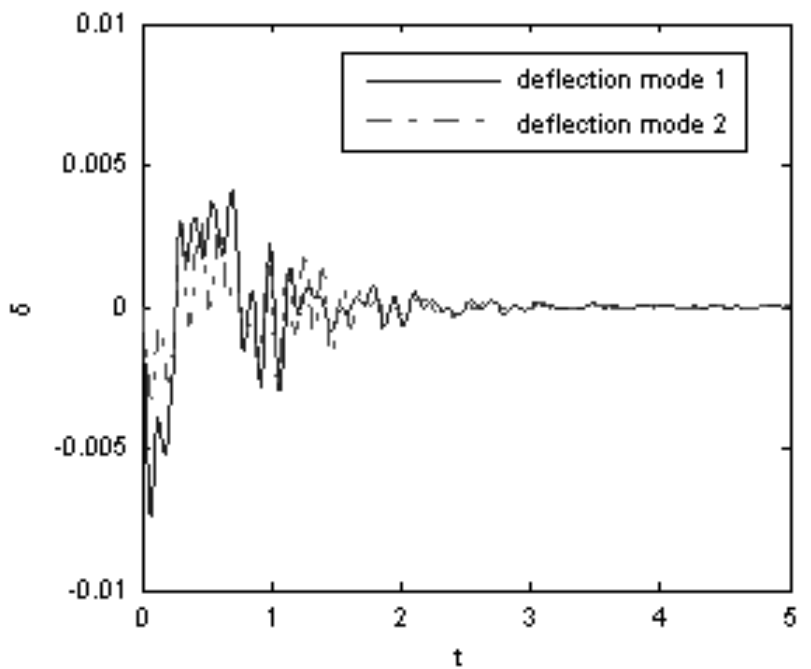

Figure 10. Deflections of first and second modes for the damped system with robust control.

For the next simulations of the system above with control law Eq. (20), we added piezoelectric actuators and sensors. We first obtained the location and sizing of the actuators solving the problem of objective function minimization, Eq. (34), using Matlab software. Figures 11 and 12 show the objective function that depends on $l_{a}$ and $x_{a}$ variables with $\alpha=300$.

The minimal value is obtained at $x_{a}=0.09 \mathrm{~m}$ and $l_{a}=0,35 \mathrm{~m}$ that respectively represents the position and sizing of piezoelectric actuator bonded on the flexible link.

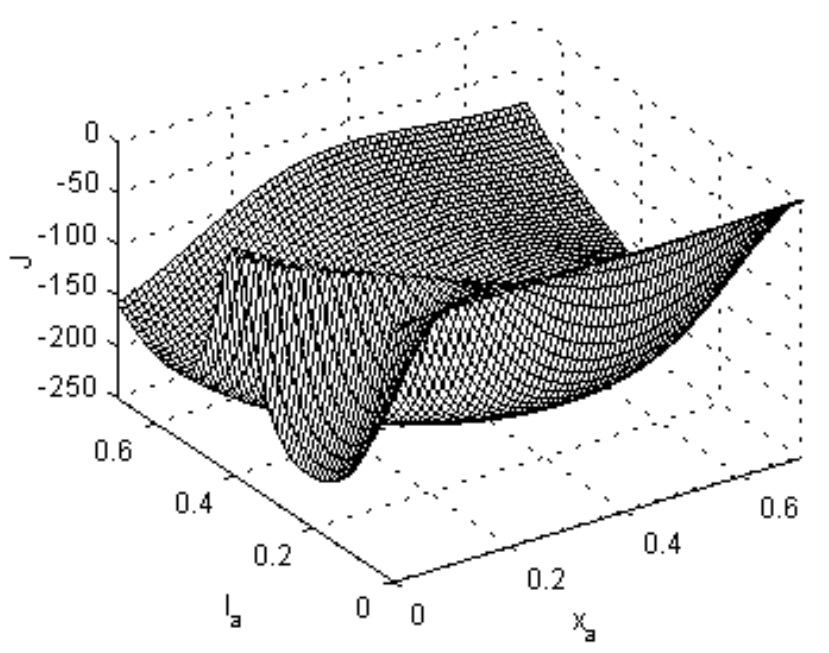

Figure 11. Dissipated energy objective function due to piezoelectric control action.

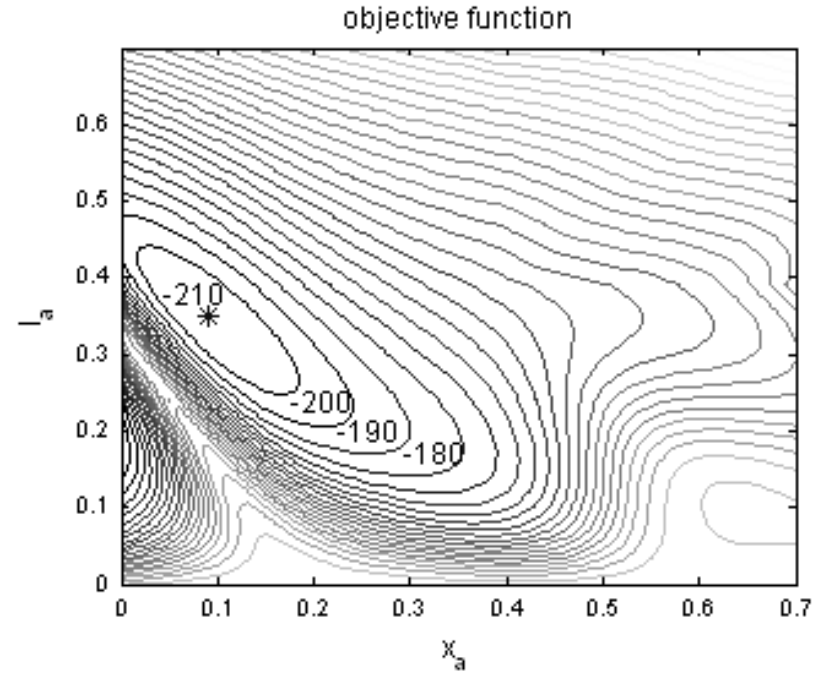

Figure 12. Contour curves of dissipated energy objective function due to piezoelectric control action.

In Figure 13, we observe that for given location and sizing of the piezoelectric actuator/sensor pairs, $J_{0}$ is a monotonous decreasing function of gain $K_{c}$. But when $K_{c}$ can be chosen very large, there are almost no variations of the value of $J$. Therefore, it is necessary to introduce the gain function $\beta_{c} k_{c}$ into the objective function Eq. (34), where $\beta_{c}$ represents a gain parameter dependent of the hardware limitations. In Fig. 14, the optimal solution for the feedback gain is $K_{c}=30$ obtained with $\beta_{c}=3$.

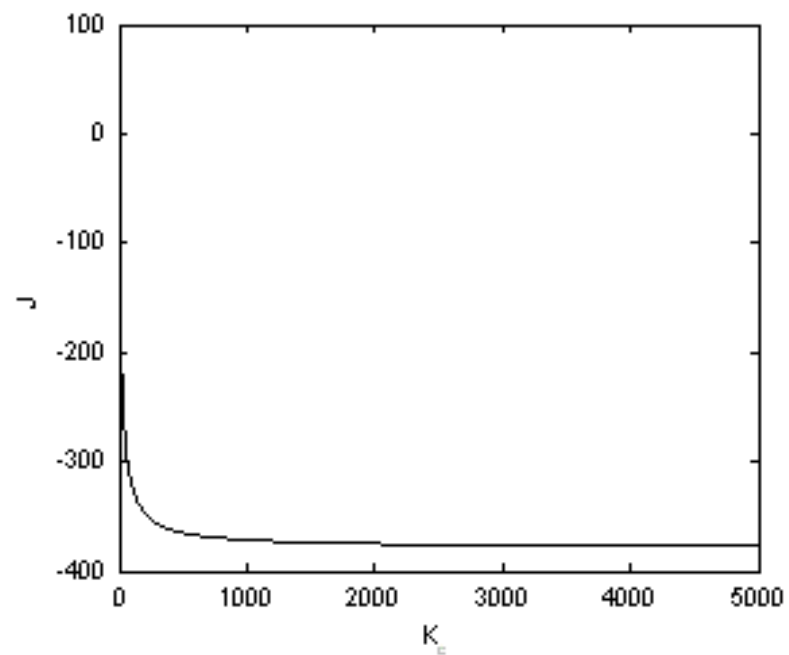

Figure 13. Objective function $\mathrm{J}$ dependency on the gain $\mathrm{K}_{\mathrm{c}}$. 


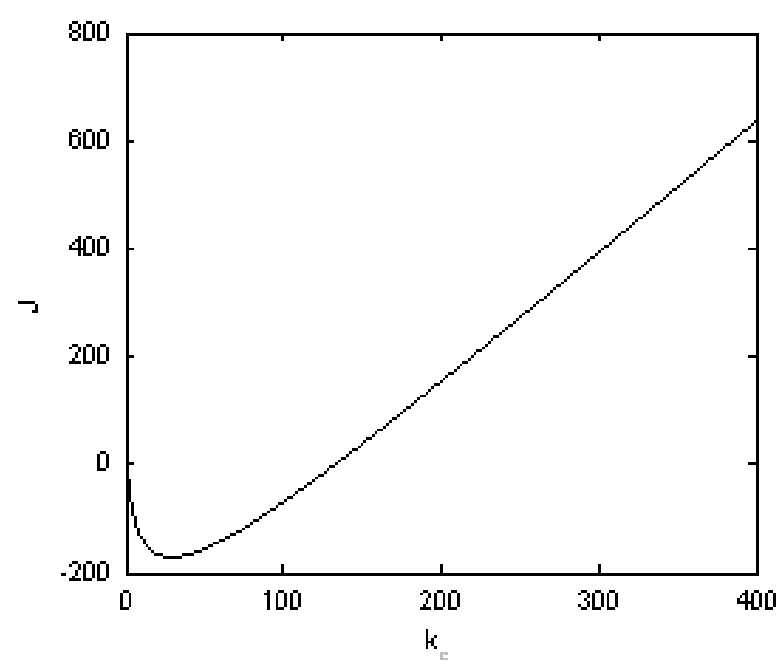

Figure 14. Objective function $\mathrm{J}$ with gain $\mathrm{B}_{\mathrm{c}} \mathrm{K}_{\mathrm{c}}$ dependency on the gain $\mathrm{K}_{\mathrm{c}}$.

Figure 15 shows a reduction on the frequency and deflection amplitude induced by the tracking control when piezoelectric actuators and sensors are added in the position $x_{a}=0.09 \mathrm{~m}$ and length $l_{a}=0.35 \mathrm{~m}$ of the piezoelectrics. It is clearly seen that the deflection frequency and amplitude is reduced by activating the piezoelectric actuator and sensor during the motion.

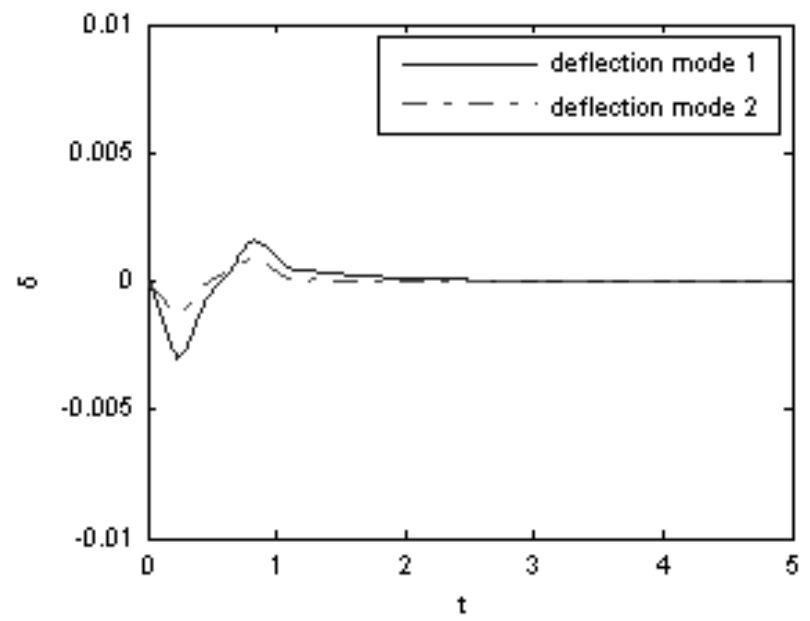

Figure 15. Deflections of first and second modes for the damped system with a piezoelectric actuator and sensor.

Figure 16 shows deflections of first and second modes for damped system with piezoelectric actuator and sensor in four different positions $x_{a}$ and lengths $l_{a}$.

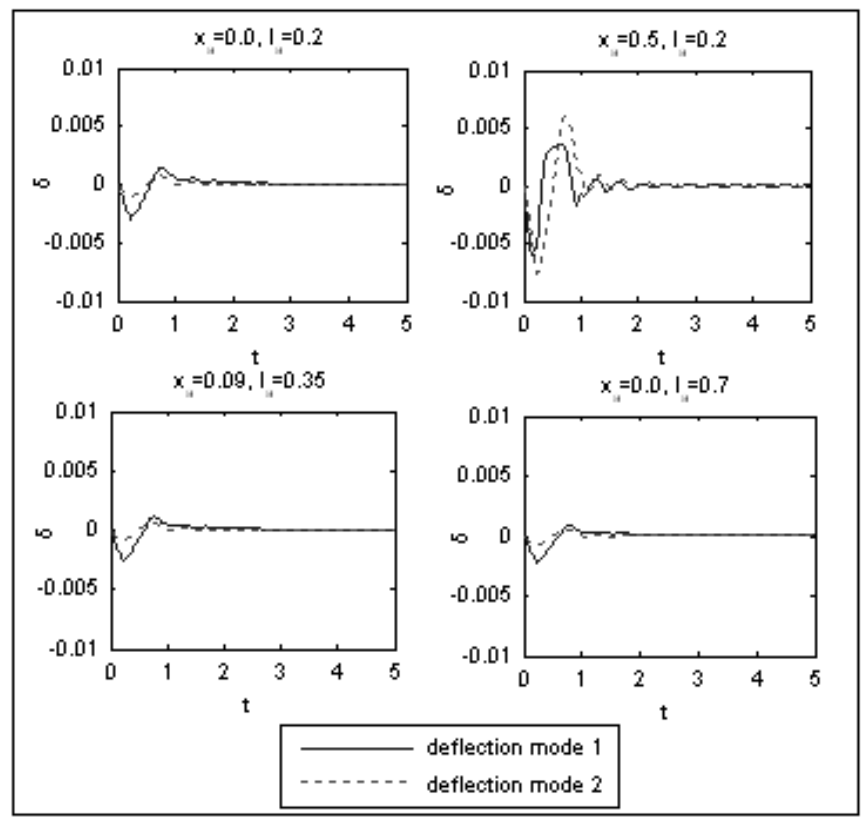

Figure 16. Deflections of first and second modes for the damped system with a piezoelectric actuator and sensor in four different positions $x_{a}$ and lengths $\mathrm{I}_{\mathrm{a}}$ : a) $\mathrm{x}_{\mathrm{a}}=0.00 \mathrm{~m}, \mathrm{l}_{\mathrm{a}}=0.2 \mathrm{~m}$; b) $\mathrm{x}_{\mathrm{a}}=0.5 \mathrm{~m}, \mathrm{l}_{\mathrm{a}}=0.2 \mathrm{~m}$; ) $\mathrm{x}_{\mathrm{a}}=0.09 \mathrm{~m}$, $\mathrm{I}_{\mathrm{a}}=0.35 \mathrm{~m}$; d) $\mathrm{x}_{\mathrm{a}}=0.0 \mathrm{~m}, \mathrm{I}_{\mathrm{a}}=0.7 \mathrm{~m}$.

As shown in Figs. 16, 17 and 18, all systems provide satisfactory tracking performance. However, the systems with piezoelectric actuator and sensor in the position and length $x_{a}=0.09 \mathrm{~m}, l_{a}=0.35 \mathrm{~m}$ and $x_{a}=0.0 \mathrm{~m}, l_{a}=0.7 \mathrm{~m}$ have a much better final tracking accuracy as seen by the performance index $\mathrm{L}$ norm Eq. (38) in Fig. 18, and a better transient as seen from performance index $\mathrm{L}^{2}$ norm Eq. (37) in Fig. 17.

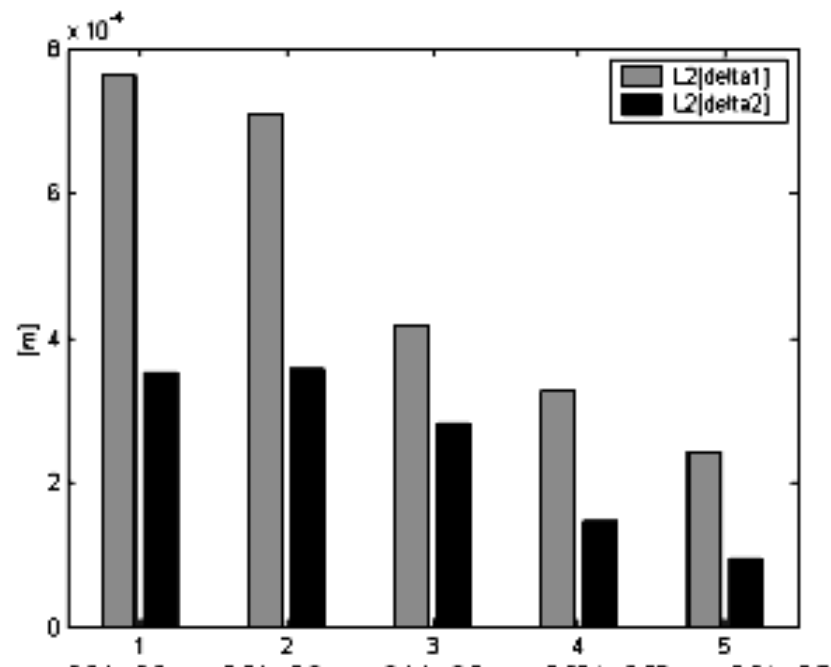

$x a=0,0|a=0,0 \quad x a=0,5 \quad l a=0,2 \quad x a=0,1 \quad l a=0,2 \quad x z=0,09| z=0,35 \quad x z=0,0 \mid a=0,7$

Figure 17. Average tracking from the performance index $L_{2}$ norm. 


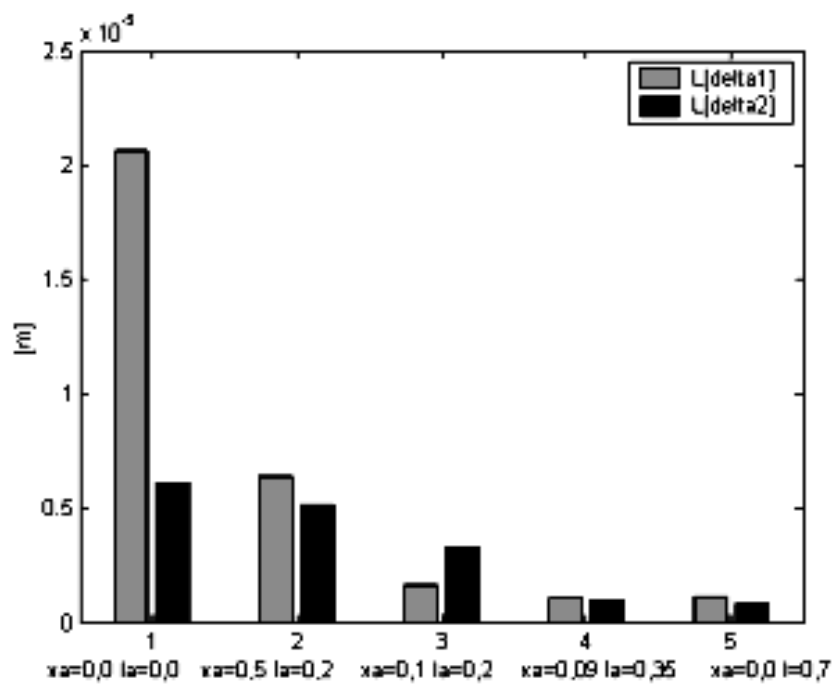

Figure 18. Average tracking during the last three seconds from the performance index $L$ norm.

It is clear that a system with a piezoelectric actuator and sensor in the position $x_{a}=0.0 \mathrm{~m}$ and length $l_{a}=0.7 \mathrm{~m}$ is the best choice, but with a piezoelectric actuator and sensor in the position and length $x_{a}=0.09 m, l_{a}=0.35 m$, respectively, it can be reduced their size and consequently the mass of the composite link with only a small loss of control performance.

Figures 19 and 20 show the torque applied to control the robot joint with and without the piezoelectric actuator, respectively. It is observed that the reduction of control effort as a high frequency of control in the second joint is reduced when the piezoelectric control is activated.

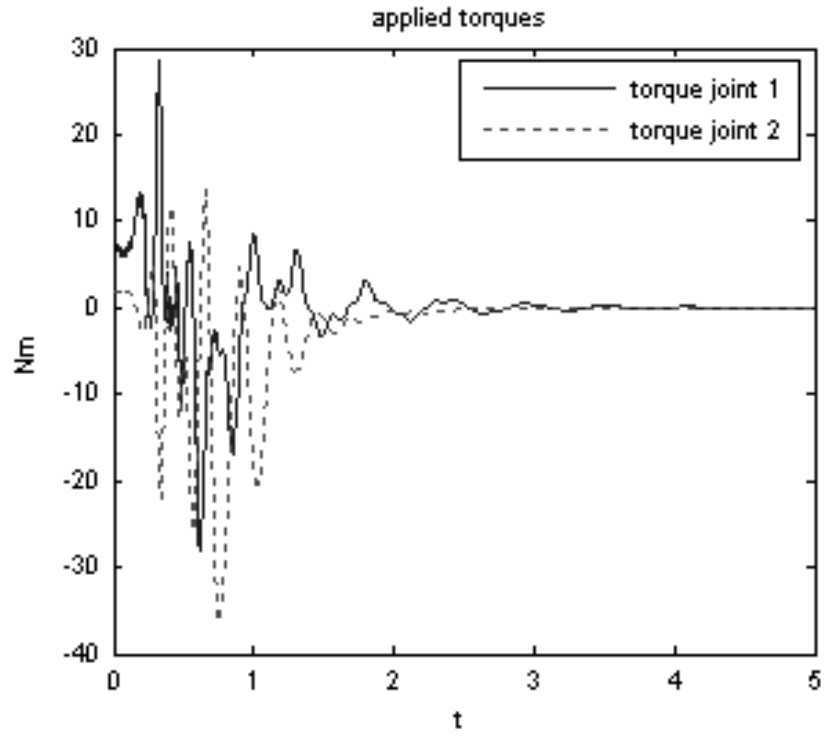

Figure 19. Applied torques without active piezoelectric actuator and sensor.

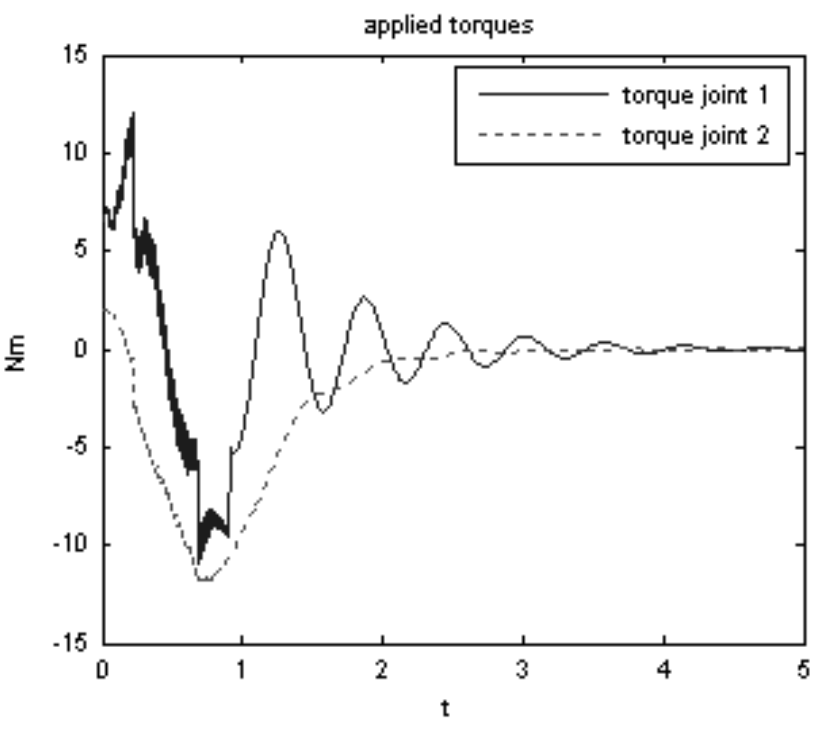

Figure 20. Applied torques with active piezoelectric actuator and sensor.

These simulation results show the advantage of combining a size measure with the control performance index to obtain the composite objective function for optimal design of link.

As a second step, a circle path is simulated in order to demonstrate favorable tracking control performance of the proposed control scheme. Figures 21 and 22 present the tracking control responses for the imposed circle trajectory without and with active piezoelectric actuator and sensor, respectively. We can distinguish the difference of control performance between two cases of the tracking error. It shows that the initial tracking error is reduced.

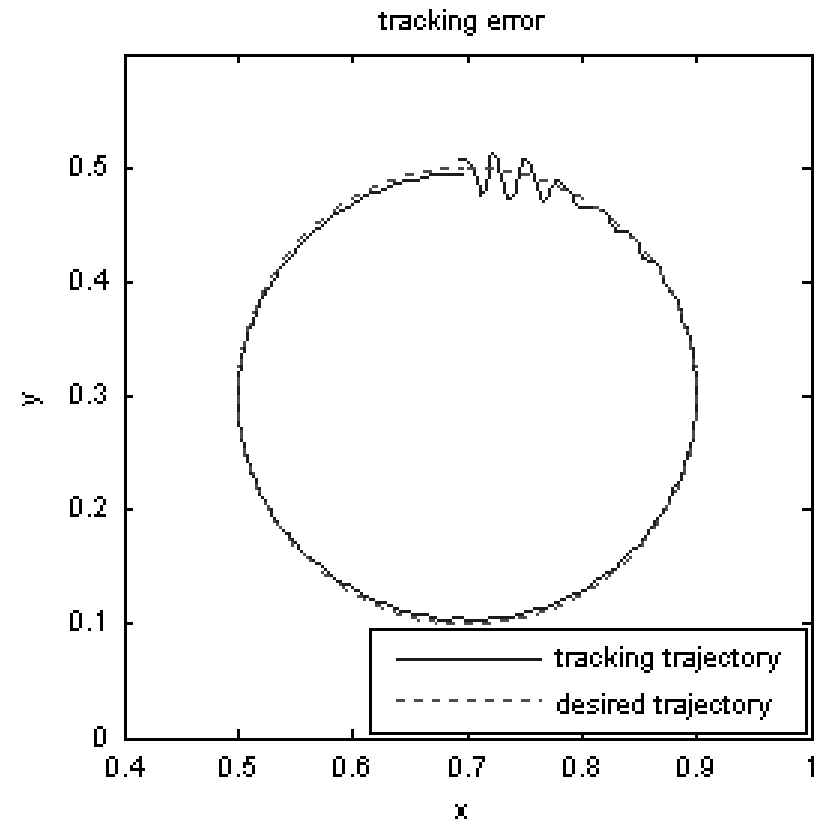

Figure 21. Tracking error of the circle path trajectory without active piezoelectric actuator and sensor. 


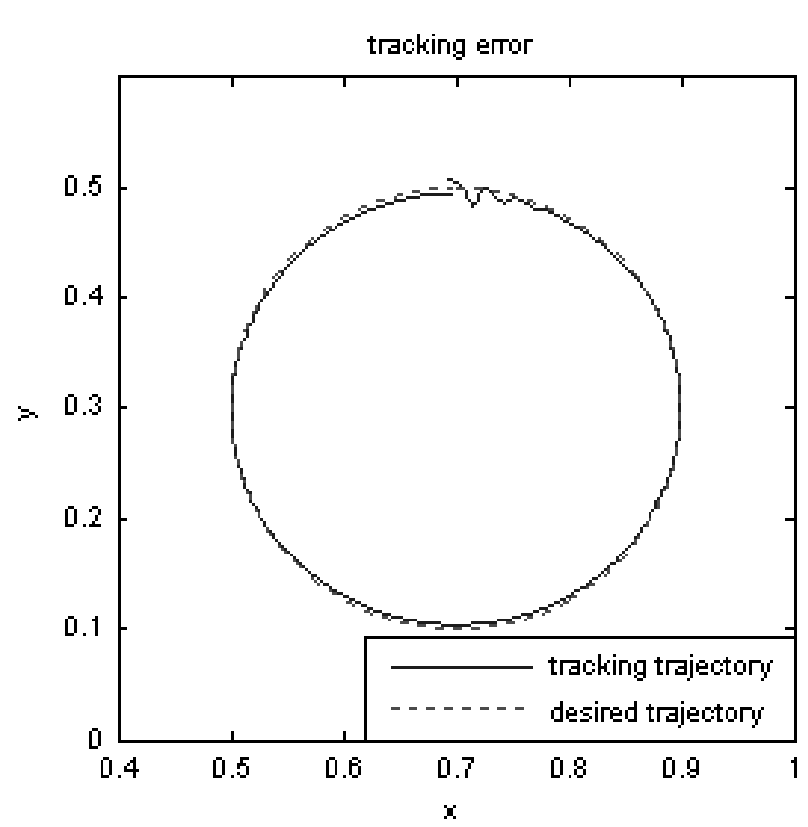

Figure 22. Tracking error of the circle path trajectory with active piezoelectric actuator and sensor.

Figures 23 and 24 present deflection control for a circle path trajectory without and with active piezoelectric actuator and sensor, respectively. It is clearly observed that the unwanted oscillations of tip deflections are favorably suppressed.

All these results support the advantage of introducing piezoelectric actuator/sensor for suppress undesirable tip deflections in the link. This control system result increases the possibilities of using the flexible link manipulator for several tasks.

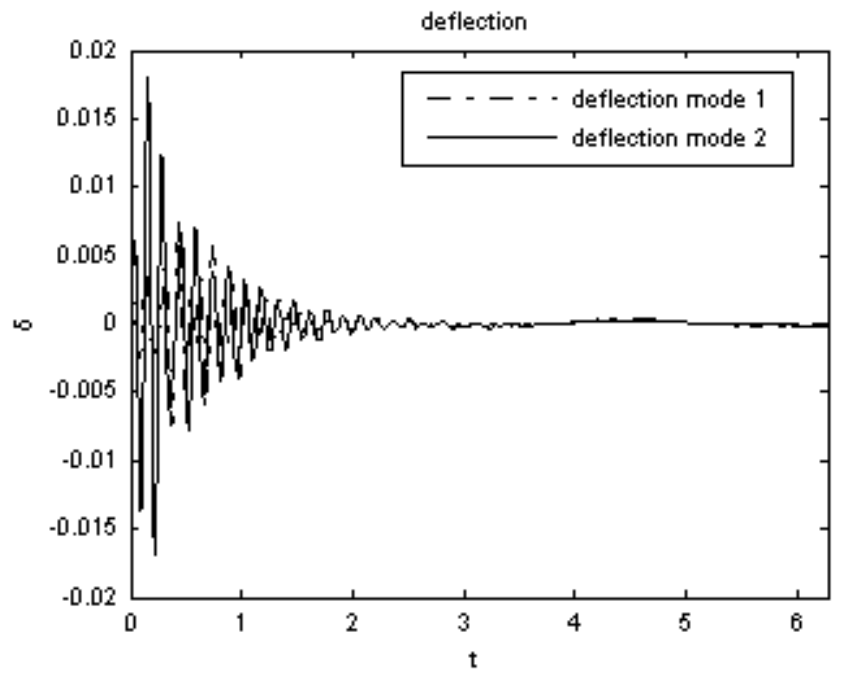

Figure 23. Deflections of first and second modes for the damped system with robust control without active piezoelectric actuator and sensor for a circle path trajectory.

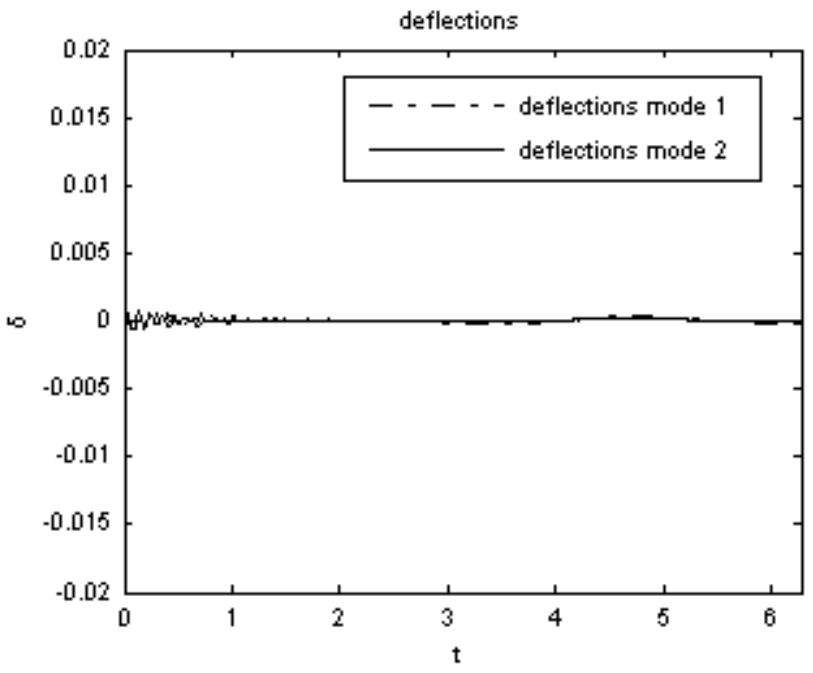

Figure 24. Deflections of first and second modes for the damped system with piezoelectric actuator and sensor for a circle path trajectory.

\section{Conclusions}

In this work we introduced a technique for tracking and vibration control of a robot with flexible links. This technique uses the motor torque for the joint angle control to control the low frequency vibrations in the robot links. Piezoelectric actuators and sensors are added to the system to control the high frequency vibrations that cannot be reduced by the motor alone. Simulation results show that this approach effectively reduces the motion induced vibration. We also introduced an optimization procedure for the sizing and position of the piezoelectric actuator and sensor, using the energy dissipated by the control in the objective function. This approach can obtain better results for motor torques that suits their control period limitation and a size reduction of the actuators and sensors sizes. This technique can be developed to build light manipulators with flexible links, while preserving the force and precision. It also reduces the energy consumption and suits the needs for aerospace systems or for tasks that demand lightness, precision and agility.

\section{Acknowledgments}

The authors would like to acknowledge the contributions of Prof. J.C.R. Claeyssen and T.T. Ruiz in the early stages of the work, as well as the useful comments and discussions by the anonymous referees of this journal, and the Department of Mathematics and Statistics, University of Caxias do Sul, for financial support.

\section{References}

Abreu, G.L.C.M., Ribeiro, J.F. and Steffen, V.J., 2003, "Experiments on optimal vibration control of a flexible beam containing piezoelectric sensors and actuators", Shock and Vibration, Vol. 10, pp. 283-300, IOS Press.

Arteaga, M.A. and Siciliano, B., 2000, "On tracking control of flexible robot arms", IEEE Transaction on Automatic Control, Vol. 45, pp 520-527.

Arteaga, M.A., 1998, "On the properties of a dynamic model of flexible robot manipuladors", ASME Jornal of Dynamics Systems, Measurement, and Control, Vol. 120, pp 8-14.

Arimoto, S., 1996, "Control Theory of Non-Linear Mechanical Systems", Oxford Clarendon Press, London.

Banks, H.T., Smith, R.C. and Wang, Y., 1996, "Smart Material Structures: Modeling, Estimation and Control”, John Wiley \& Sons, Paris.

Book, W.J., 1984, "Recursive lagrangian dynamics of flexible manipulator arms", Int. J. Robotics Res., Vol. 3, No. 3, pp. 87-101. 
Bottega, V. Pergher, R., Molter, A. and Fonseca, J.S.O., 2007, "Modelagem, controle e simulação de manipuladores robóticos com braços flexíveis de geometria irregular", In: CILAMCE Iberian Latin American Congress on Computational Methods in Engineering, Porto, Portugal.

Cho, S.B., Cho, S.S., Shin, H.C. and Kim, H.K., 1999, "Quantitative feedback theory control of a single-link flexible manipulator featuring piezoelectric actuator and sensor", Smart Material Structures, Vol. 8, pp. 338-349.

Choi, S. and Shin, H., 1996, "A hibrid actuator scheme for robust position control of a flexible single-link manipulator", Journal of Robotic Systems, Vol. 13, No. 6, pp. 359-370.

Copetti, R.D., Claeyssen, J.C.R. and Tsukazan, T., 2007, "Modal formulation of segmented Euler-Bernoulli beams", Mathematical Problems in Engineering, Vol. 2007, Article ID 36261, 18 p.

Crawley, E.F. and De Luis, J., 1987, "Use of piezoelectric actuators as elements of intelligent structures", AIAA Journal, Vol. 25, pp. 1373-1385.

De Luca, A., Lucibello, P. and Nicolo, F., 1988, "Automatic symbolic modeling and nonlinear control of robots with flexible links". Proc. IEEE Work on Robot Control, Oxford, UK, Apr., pp. 62-70.

De Luca, A., Lanari, L., Lucibello, S. and Panzieri, S., 1990, "Control experiments on a two-link robot with a flexible forearm", 29th IEEE Conf. Decision and Control, Honolulu, HI, pp 5-7.

Denoyer, K.K. and Kwak, M.K., 1996, "Dynamic modelling and vibration suppression of a slewing structure utilizing piezoeletric sensor and actuators", Journal of Sound and Vibration, Vol. 189, No. 1, pp. 13-31.

Dhuri, K.D. and Seshu, P., 2006. "Piezoactuator placement and sizing for good control effectiveness and minimal change in original system dynamics". Smart Mater. Struct, Vol. 15, pp. 1661-1672.

Gabbert, U. and Schulz, I., 1996, "Optimal placement of piezoelectric actuators in vibration control of adaptative structures", Proc. ASME Int. Mech. Eng. Congress and Exposition, DE 93, pp. 271-277.

Kermani, M.R., Moallem, M. and Patel, R.V., 2004, "Parameter selection and control design for vibration suppression using piezoelectric transducers", Control Engineering Practice, Vol. 12, pp. 1005-1015.

Kim, H.K., Choi, S.B. and Thompson, B.S., 2001, "Compliant control of a two-link flexible manipulator featuring piezoelectric actuators", Mechanism and Machine Theory, Vol. 36, pp. 411-424.

Knani, J., 2002, "Dynamic modelling of flexible robotic mechanisms and adaptive robust control of trajectory computer simulation - Part I", Applied Mathematical Modelling, Vol. 26, pp. 1113-1124.
La Salle, J.P. and Lefschetz, S., 1961, “Stability by Lyapunov's Direct Method", Academic Press, New York.

Li, Y., Onoda, J. and Minesugi, K., 2002, "Simultaneous optimization of piezoelectric actuator placement and feedback for vibration suppression", Acta Astronautica, Vol. 50, No. 6, pp. 335-341.

Liu, X. and Begg, D.W., 2000, "On simultaneous optimization of smart structures-part I", Theory, Computer Methods in Applied Mechanics Engineering, Vol. 184, pp. 15-24.

Meirovitch, L., 1967. "Analitical Methods in Vibration", Macmillan, New York.

Mohamed, Z., Martins, J.M., Tokhi, M.O., Costa, J.S. and Botto, M.A., 2005. "Vibration control of a very flexible manipulator system", Control Engineering Practice, Vol. 13, pp. 267-277.

Naidu, D.S., 2003. "Optimal Control System”, CRC Press, New York.

Shin, H. and Choi, S., 2001, "Position control of a two-link flexible manipulator featuring piezoelectric actuators and sensors", Mechatronics, Vol. 11, pp. 707-729.

Siciliano, B. and Valavanis, K.P., 1998, "Control Problems in Robotics and Automation", Springer-Verlag, London.

Sun, D., Mills, J.K., Shan, J. and Tso, S.K., 2004 "A PZT actuator control of a single-link flexible manipulator based on linear velocity feedback and actuator placement", Mechatronics, Vol. 14, pp. 381-401.

Truhar, N., 2004, "An efficient algorithm for damper optimization for linear vibrating systems using Lyapunov equation", Journal of Computational and Applied Mathematics, Vol. 172, pp. 169-182.

Truhar, N. and Veselić, K., 2004, "On some properties of the Lyapunov equation for damped systems", Mathematical Communications, Vol. 9, No. 2 , pp. 189-197.

Tsukazan, T., 2005, "The use of a dynamical basis for computing the modes of a beam system with a discontinuous cross-section", Journal of Sound and Vibration, Vol. 281, no. 3-5, pp. 1175-1185.

Yao, B. and Tomizuca, M., 1996, "Smooth robust adaptive sliding mode control of manipulators with guaranteed transient performance", Journal of Dynamic Systems, Measurement and Control, Vol. 118, pp.764-775.

Zhang, X., Xu, W., Nair, S.S. and Chellaboina, V., 2005, "PDE modeling and control of a flexible two-link manipulator", IEEE Transaction on Control Systems Technology, Vol. 13, n. 2, March. 